\title{
No link to cardiac valvulopathy was seen in cabergoline treated patients
}

\author{
K Dharshini ${ }^{1}$, Noel Somasundaram² ${ }^{2}$ H M D P K Senevirathna ${ }^{3}$, S Raj Kumar ${ }^{4}$ \\ Sri Lanka Journal of Diabetes, Endocrinology and Metabolism 2012; 2: 69-72 \\ (Index words: prolactinoma, cabergoline, valvulopathy)
}

\begin{abstract}
Objective: To evaluate the association between use of cabergoline in patients with prolactinoma and risk of developing clinically relevant cardiac valvular disease.

Method: This was a descriptive cross sectional study conducted at Endocrinology and Pituitary clinics at National Hospital of Sri Lanka. Patients with macro and micro prolactinoma who were on medical therapy with cabergoline for more than 24 weeks duration were selected for the study. Patients with pre existing congenital valvular heart disease, history of rheumatic heart disease and ischemic heart disease were excluded from the study. All the included patients were evaluated with $2 \mathrm{D}$ echocardiogram for the presence and degree of valvulopathy.
\end{abstract}

Results: Out of 45 patients included in this study, 12 (26.6\%) had mild valvular regurgitation. Twenty four patients received more than the median cumulative dose of $104 \mathrm{mg}$ and the prevalence of valvulopathy was not significantly different (Chi-Squared test; $p=0.34$ ) from those receiving less than the median cumulative dose. Similarly 23 patients received treatment longer than the median duration of 48 months and the prevalence of valvulopathy was not significantly different (Chi-square test; $p=0.92$ ) from those received less than the median duration.

Conclusion: Our study shows no significant association between the cumulative dose and the duration of cabergoline therapy with the development of valvulopathy among patients with prolactinoma.

\section{Introduction}

Medical therapy has become the main treatment modality in treating prolactinomas and dopamine agonists such as cabergoline, quinagolide and bromocirptine are the commonly used drugs in clinical practice. Due to the convenience of weekly dosing and lesser side effects compared to the other dopamine agonist, cabergoline has become the drug of choice for most of the patients. Cabergoline is efficacious and well tolerated and has shown to reduce the prolactin level as well as the pituitary adenoma volume. Cabergoline has a high affinity for 5-hydroxytryptamine receptor 2B (HTR2B) located on heart valves. There is a suggestion that activation of these receptors might lead to mitogenesis and fibroblast proliferation in cardiac valves. Two population-based studies in patients with Parkinson's disease showed an increased risk of valve regurgitation after treatment with high cumulative dose of pergolide and cabergoline $(1,2)$. This raises serious concerns with respect to the safety of the long-term use of cabergoline in the treatment of prolactinoma.

There were eleven cross-sectional studies, which have been published recently, with a total of 795 patients with prolactinoma (3-13). Five of those studies did not show any association between the treatment with cabergoline and clinically relevant valvular regurgitation during 45-79 months of therapy (3-7). However, the use of cabergoline was associated with an increased prevalence of moderate tricuspid regurgitation in one study (8). Furthermore, the use of cabergoline was associated with increased frequencies of valvular thickening, calcifications and increased mitral tenting area (9). At present, the clinical relevance of these findings is still uncertain, but concern is raised with respect to the safety of the use of cabergoline in the long-term treatment of prolactinomas. There seems to be an individual susceptibility of the HTR2B on cardiac valves for the agonist activity or affinity of cabergoline since polymorphisms of the serotonin

${ }^{1}$ Senior Registrar in Endocrinology, National Hospital of Sri Lanka,Colombo, ${ }^{2}$ Consultant Endocrinologist, National Hospital of Sri Lanka,Colombo, ${ }^{3}$ Senior Registrar in Cardiology, National Hospital of Sri Lanka, Colombo ${ }^{4}$ Trainee, Medical Administration, PGIM. 
receptor have been described (14-16). Hence there is a need for assessing the genetic susceptibility of developing clinically relevant valvular disease with cabergoline treatment in our population.

\section{Objectives}

Our objective was to evaluate the association between use of cabergoline in patients with prolactinoma and risk of developing clinically relevant valvular disease.

\section{Methodology}

\section{Study design, setting and study population}

This was a descriptive cross sectional study conducted in the Endocrinology and Pituitary clinics at National Hospital of Sri Lanka. All diagnosed patients with micro and macro prolactinoma who were on medical therapy with cabergoline for more than 24 weeks duration at the date of data collection were included in this study. Age group of this sample was 21 to 54 years.

Diagnosed patients with pre existing congenital valvular heart disease, history of rheumatic heart disease and ischemic heart disease were excluded from the study.

Data collection was carried out for three months starting from 1st of March, 2012. A data sheet was used for data collection and data gathered from clinic records with regard to the following aspects. Age, sex, pre treatment body weight, pre treatment prolactin levels and other hormonal assessment in standard laboratory units (thyroid stimulating hormone, freeT4, serum cortisol, follicular stimulating hormone, luteinizing hormone) were recorded. Diagnosis (macroprolactinoma, microprolactinoma), dose of drug, frequency of administration and duration of therapy were also recorded.

The patients fulfilling the inclusion criteria were investigated with 2D echocardiogram by the same operator (a Senior Registrar in Cardiology), at National Hospital of Sri Lanka. Following aspects were assessed; the presence of valvular regurgitation, the degree of regurgitation (rated in mild, moderate or severe regurgitation according to the recommendations of the American Society of Echocardiography), and the presence of morphological changes such as thickening and/or calcification. Data with regard to post treatment outcome such as post treatment prolactin levels in standard laboratory units and post treatment body weight were gathered.

\section{Statistical analysis}

Analysis of data was carried out using SPSS 18 version. Mean, median values and standard deviation were estimated for each continuous variable, such as age, body weight, prolactin levels, duration of therapy and cumulative dose; while proportions were estimated for categorical variables. Chi sqaure value was used to compare proportions while Student's t test was used to compare continuous variables. $\mathrm{p}<0.05$ was considered as the level of significance.

\section{Results}

From a total of 58 patients, 45 patients (35, 77.7\% females) were included in the study. The mean age of the participants was $37.4(+/-9.1)$ years, the mean pre treatment body weight was $62.82(+/-12.4) \mathrm{kg}$ and the mean pre treatment prolactin levels were $16074(+/-9221) \mathrm{miu} / \mathrm{L}$. The median cumulative dose of cabergoline used was $104 \mathrm{mg}$ and the median duration of treatment was 48 months. Twelve patients (26.6\%) had mild regurgitation (Table 1). Twenty four patients received more than the median cumulative dose of $104 \mathrm{mg}$ and the prevalence of valvulopathy was not significantly different (Chi-Squared test; $\mathrm{p}=0.34$ ) from those receiving less than the median cumulative dose. Similarly 23 patients received treatment longer than the median duration of 48 months and the prevalence of valvulopathy was not significantly different (Chi-square test; $\mathrm{p}=0.92$ ) from those received less than the median duration (Table 2 and Figure 1). The mean post treatment prolactin level was $836(+/-216) \mathrm{miu} / \mathrm{L}$ and the reduction of the prolactin levels from the baseline level due to cabergoline therapy was significant (paired $t$ test; $\mathrm{p}=0.004)$.

\section{Discussion}

We found no clinically significant cardiac valve disease among patients treated with cabergoline for prolactinoma and this is similar to the outcome of previously conducted cross sectional case controlled studies.

Lancellotti and colleagues (9) undertook a readerblind, cross-sectional study of 102 outpatients who received cabergoline for the treatment of prolactinoma or idiopathic hyperprolactinaemia. The median cumulative dose was $204 \mathrm{mg}$ and the median duration of therapy was 79 months. The frequency of moderate or severe valvular regurgitation did not differ significantly between the treatment and the control groups. Several other studies confirm these findings (3-7). In contrast, Colao and coworkers (8) compared 50 patients receiving a median cumulative dose of cabergoline $280 \mathrm{mg}$ over a median period of 74 months with 50 age and sex-matched healthy controls and 20 untreated patients. The frequency of aortic regurgitation or mitral regurgitation did not differ significantly between the groups in this study. However, the prevalence of tricuspid regurgitation was significantly higher in those receiving more than the median cumulative dose than in those given less than the median cumulative dose. 
Table 1. Age category, diagnosis and proportions of valvulopathy in the sample patients

\begin{tabular}{lllll}
\hline & & Males & Females & Total \\
\hline Age category (years) & $21-30$ & 2 & 10 & $12(26.6 \%)$ \\
& $31-40$ & 4 & 15 & $19(42.2 \%)$ \\
& $41-50$ & 3 & 8 & $11(24.4 \%)$ \\
Diagnosis & More than 50 & 1 & 2 & $3(6.6 \%)$ \\
& Macroprolactinoma & 10 & 15 & $25(55.5 \%)$ \\
Presence of valvulopathy & Microprolactinoma & 0 & 20 & $20(44.5 \%)$ \\
& & & & $12(26.6 \%)$ \\
& MR & 4 & 2 & 3 \\
Absence of valvulopathy & PR & 3 & 2 & 1 \\
& MR and TR & 1 & 1 & 2 \\
\hline
\end{tabular}

MR - Mitral Regurgitation, TR - Tricuspid Regurgitation, PR - Pulmonary Regurgitation.

Table 2. Prevalence of valvulopathy with cumulative dose and duration of therapy

\begin{tabular}{lllll}
\hline & & Valvulopthy & No valvulopathy & Total \\
\hline Cumulative dose of cabergoline & Less than median dose & $7(33.3 \%)$ & $14(66.7 \%)$ & 21 \\
& More than median dose & $5(20.8 \%)$ & $19(79.2 \%)$ & 24 \\
Duration of therapy & Less than median duration & $6(27.2 \%)$ & $16(72.8 \%)$ & 22 \\
& Longer than median duration & $6(26.1 \%)$ & $17(73.9 \%)$ & 23 \\
\hline
\end{tabular}

This shows the median cumulative dose (the horizontal line in the box), 25th to 75th percentiles and the range excluding outliers (5 and 34) among patients with and without valvulopathy.

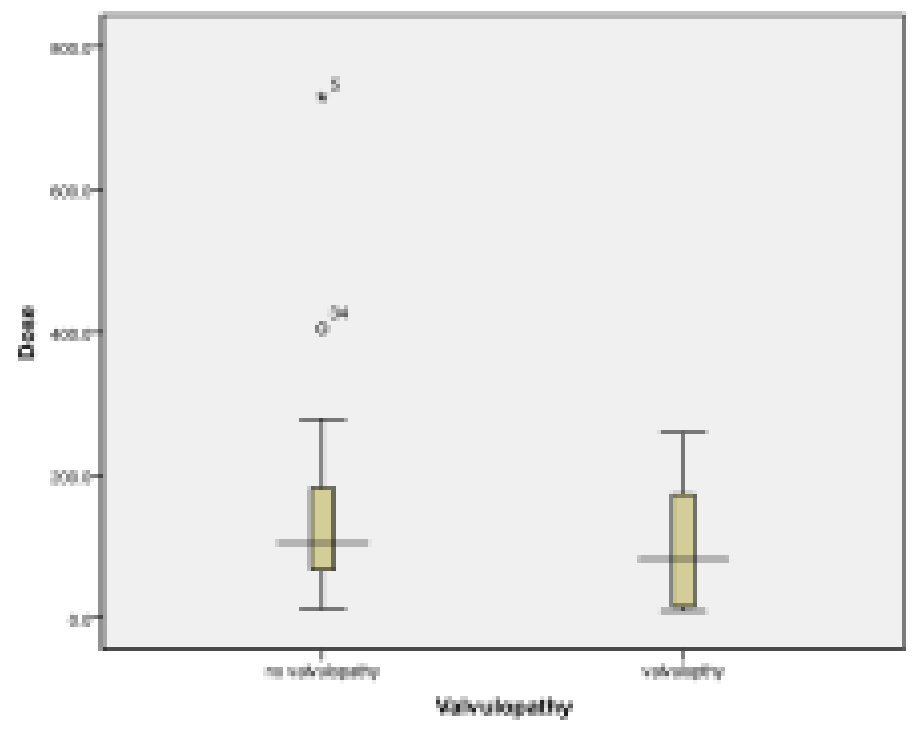

Figure 1. Stem and leaf plot. 
Our study showed a prevalence of $26.6 \%$ mild valvular regurgitation and there was no significant association with the cumulative dose of cabergoline and the duration of therapy. Small sample size was one of the main limitations of our study and the lack of a baseline (pre treatment) echocardigraphy was another drawback. However, this does not invalidate our findings and prospective studies with sufficient power are required to investigate the true prevalence of cardiac valve disease related to the modest doses of cabergoline that are normally used in patients with prolactinoma.

\section{References}

1. Zanettini R, Antonini A, Gatto G, Gentile R, Tesei S, Pezzoli G. Valvular heart disease and the use of dopamine agonists for Parkinson's disease. N Engl J Med 2007; 356: 39-46.

2. Schade R, Andersohn F, Suissa S, Haverkamp W, Garbe E. Dopamine agonists and the risk of cardiac-valve regurgitation. N Engl J Med 2007; 356: 29-38.

3. Bogazzi F, Buralli S, Manetti L, Raffaelli V, Cigni T, Lombardi $\mathrm{M}$, et al. Treatment with low doses of cabergoline is not associated with increased prevalence of cardiac valve regurgitation in patients with hyperprolactinaemia. Int J Clin Pract 2008; 62: 1864-9.

4. Vallette S, Serri K, Rivera J, Santagata P, Delorme S, Garfield $\mathrm{N}$, et al. Longterm cabergoline therapy is not associated with valvular heart disease in patients with prolactinomas. Pituitary 2009; 12: 153.

5. Herring N, Szmigielski C, Becher H, Karavitaki N, Wass JA. Valvular heart disease and the use of cabergoline for the treatment of prolactinoma. Clin. Endocrinol (Oxf) 2009;70: 104-8.

6. Lafeber $\mathrm{M}$ et al. Absence of major fibrotic adverse events in hyperprolactinemic patients treated with cabergoline. Eur $J$ Endocrinol 2010; 162: 667-5.
7. Tan $\mathrm{T}$ et al. Assessment of cardiac valve dysfunction in patients receiving cabergoline treatment for hyperprolactinaemia. Clin Endocrinol (Oxf) 2010; 73: 369-74.

8. Colao A, Galderisi M, Di Sarno A, Pardo M, Gaccione M, D’Andrea $M$, et al. Increased prevalence of tricuspid regurgitation in patients with prolactinomas chronically treated with cabergoline. J Clin Endocrinol Metab 2008; 93: 3777-84.

9. Lancellotti P, Livadariu E, Markov M, Daly AF, Burlacu MC, Betea D, et al. Cabergoline and the risk of valvular lesions in endocrine disease. Eur J Endocrinol 2008; 159: $1-5$.

10. Kars M, Delgado V, Holman ER, Feelders RA, Smit JW, Romijn JA, et al. Aortic valve calcification and mild tricuspid regurgitation, but no clinical heart disease after 8 years of dopamine agonist therapy for prolactinoma. J Clin Endocrinol Metab 2008; 93: 3348-56.

11. Wakil A, Rigby A, Clark A, Atkin S. Low dose cabergoline for hyperprolactinaemia is not associated with clinically significant valvular heart disease. Eur J Endocrinol 2008; 159: R11-4.

12. Devin JK, Lakhani VT, Byrd BF. 3rd and Blevins LS. Jr. Prevalence of valvular heart disease in a cohort of patients taking cabergoline for management of hyperprolactinemia. Endocr Pract 2008; 14: 672-7.

13. Nachtigall LB et al. Gender effects on cardiac valvular function in hyperprolactinaemic patients receiving cabergoline: a retrospective study. Clin Endocrinol (Oxf) 2010; 72: 53-8.

14. Arranz MJ, de Leon J. Pharmacogenetics and pharmacogenomics of schizophrenia: a review of last decade of research. Mol Psychiatry 2007; 12: 707-47.

15. Malhotra AK, Lencz T, Correll CU, Kane JM. Genomics and the future of pharmacotherapy in psychiatry. Int Rev Psychiatry 2007; 19: 523-30.

16. Lung B, Vahanian A. Epidemiology of valvular heart disease in adults. Nat Rev Cardiol 2011; 10: 1038. 